\title{
Genetic Variability Studies for Yield and Yield Attributing Traits in Brinjal (Solanum melongena L.)
}

\author{
P.G. Shruti ${ }^{*}$, C.N. Hanchinamani, N. Basavaraja, \\ M.S. Kulkarni, S. Cholin and M.H. Tatagar
}

Kittur Rani Channamma, College of Horticulture Arabhavi - 591 218, India

University of Horticultural Sciences, Bagalkot, India

*Corresponding author

\begin{tabular}{|l|}
\hline Ke y w o r d s \\
Brinjal (Solanum \\
melongena L.), \\
Pusa Shymala, \\
Genetic variability \\
\hline Article Info \\
\hline $\begin{array}{l}\text { Accepted: } \\
\text { 17 June } 2019 \\
\text { Available Online: } \\
\text { 10 July } 2019\end{array}$ \\
\hline
\end{tabular}

\section{Introduction}

Brinjal (Solanum melongena L.) is an important and principle vegetable crop of India. It belongs to the family Solanaceae and is native of Indo-Berma region. Solanum is a very large and important genus comprising
The investigation on "Phenotypic evaluation and genetic variability studies in brinjal (Solanum melongena L.)" was undertaken during kharif season of 2017. Sixty brinjal genotypes were evaluated in randomized block design with two replications in vegetable science block, Dept. of Vegetable Science, Kittut Rani Channamma College of Horticulture, Arabhavi under the University of Horticultural Sciences, Bagalkot. In the study, analysis of variance revealed highly significant differences among genotypes for most of characters studied. The growth parameters viz., high phenotypic coefficient of variation $(>20 \%)$ with moderate genotypic coefficient of variation (11$20 \%$ ) was noticed in plant height at 90 DAT, number of primary branches per plant at 90 DAT, stem girth at 90 DAT and leaf area. Fruit weight, number of fruits per cluster and fruit girth had reported moderate phenotypic coefficient of variation $(>20 \%)$ with genotypic coefficient of variation, while number of fruits per plant, fruit length, fruit yield per plant and phenols showed high phenotypic coefficient of variation with genotypic coefficient of variation. Phenols showed high phenotypic coefficient of variation with genotypic coefficient of variation $(>20 \%)$ depicting the additive component is predominant. High heritability $(>60 \%)$ estimates along with high GAM $(>20 \%)$ was also noted in phenols. Plant height was found maximum in Acc-113 $(64.90 \mathrm{~cm})$, primary branches per plant 3.10 (Acc-65) to 7.00 (Pusa Shyamala), Minimum number of days to fifty \% flowering (55.00) was observed in HUB - 23, IC 354597 recorded minimum fruit weight (73.00 g) while, maximum in Acc - 597 (174.30 g). HUB -13 recorded maximum number of fruits per plant (21.60) while, CO-2 with minimum number of fruits (7.40). Maximum fruit yield per plant was noted 2.67 $\mathrm{kg}$ in HUB - 13. Among the genotypes studied, phenols ranged from 75.98 (IC 354597) to 267.60 (Pusa Shymala). These results indicated the existence of sufficient variability in genetic stock studied and the environmental role is negligible. Hence, there is ample scope for improving these characters with direct selection in further breeding programme. 
grows under wide range of soil and climatic conditions. There is an increasing demand for its varieties for different culinary purposes. It is considered as brain food and poor man's caviar. The immature fruit is primarily used as cooked vegetable and utilized in the preparation of various dishes like sliced bhaji, stuffed curry, bertha, chutney, vangibath and pickles in different parts of the world. Brinjal is quite high in nutritive value and it is an important source of carbohydrate $(4.0 \mathrm{~g})$, protein $(1.4 \mathrm{~g})$, fibre $(1.3 \mathrm{~g})$, vitamin A (124 $\mathrm{IU})$, phosphorus $(47 \mathrm{mg})$, potassium $(2.0 \mathrm{mg}$ ) and iron $(0.3 \mathrm{mg})$ and recommended for diabetes, asthma, cholera, bronchitis and it protects the brain cell membranes from damage. Brinjal has more regional preferences for specificity of fruits trait ranging from round to long fruit with green, purple, pink, white and stripped multicolours. Considering the potentiality of this crop, there is a prime need for improvement and to develop varieties suited to specific agro-ecological conditions and also for specific use. The role of genetic variability in crops is of paramount importance in selecting the best genotypes for making rapid improvement in yield and related characters as well as to select the most potential parents for making the hybridization programme successful. The success of any crop improvement programme largely depends upon the nature and magnitude of the genetic variability existing in breeding material with which plant breeder is working. The phenotypic expression of the plant character is mainly controlled by the genetic makeup of the plant and environment, in which it is grown and the interaction between the genotype and environment. Further, the genetic variance of any quantitative trait is composed of additive variance (heritable) and non-additive variance (non heritable), which include dominance and epistasis (non-allelic interaction). Therefore, it becomes necessary to partition the observed phenotypic variability into its genotypic (partly heritable) and environmental (non heritable) components with suitable parameters, such as phenotypic and genotypic coefficient of variation and heritability in broad sense. Further, genetic advance can be used to predict the efficiency of selection. Effectiveness of selection directly depends on the amount of heritability and genetic advance as per cent of mean for that character. With this background, the present investigation to evaluate the phenopypic traits and genetic variability for different characters to understand the scope for selection was undertaken.

\section{Materials and Methods}

Research was conducted in KRCCH Arabhavi, with 60 genotypes in Randomized Complete Block Design with two replication. Seeds were sown in portrays which were filled with the mixture of cocopeat and vermicompost during May 2017. These protrays were covered with black polythene to build up humidity for better and early germination of seeds. After germination, polythene cover was removed and watering was done either in the morning or evening hours. The 19:19:19 nutrient spray was taken to seedlings @ $0.5 \mathrm{gm} /$ litre of water at 25 days after sowing and again this was repeated after 10 days. Triazophos spray @ $1.5 \mathrm{ml} /$ litre of water was taken to protect from leaf miner incidence. Then the seedlings after 35 days were transplanted at spacing of $75 \mathrm{~cm} \times 60 \mathrm{~cm}$ to the main field.

\section{Results and Discussion}

A virtual and significant results were noticed for growth and yield parameters. Plant height at 90 DAT varied from $31.16 \mathrm{~cm}$ (HUB-3) to $64.90 \mathrm{~cm}$ (Acc-113) with an average mean of $39.86 \mathrm{~cm}$. Low GCV (8.91 \%) and moderate PCV (19.00 \%) were associated with low heritability and GAM (21.98 \% and $8.60 \%)$ with GA 3.43. Number of primary branches 
per plant at 90 DAT ranged from 3.10 (Acc 65) to 7.00 (Pusa Shyamala) with an average mean of 5.23. GV and PV were 0.50 and 0.89 respectively. This trait recorded moderate GCV and PCV (13.59 \% and $18.05 \%$ respectively) along with moderate heritability $(56.75 \%)$, low GAM $(21.10 \%)$ and GA of 1.10. L - 3267 with highest stem girth (1.65 $\mathrm{cm})$, along with an average mean of $1.02 \mathrm{~cm}$. GV and PV were 0.03 and 0.07 respectively. Moderate GCV (17.80 \%) and high PCV (26.36\%) was noticed along with moderate heritability (45.61\%), low GAM 2(4.77\%) and GA $(0.25)$ for this character. Leaf area of $459.75 \mathrm{~cm}^{2}$ (HUB - 22) and Low GCV (19.22 $\%)$ and high PCV (35.69 \%) were associated with low heritability (29.02 \%), low GAM $(21.33 \%)$ and GA (64.57). High heritability in the traits indicated the predomonance of additive genetic components and direct selection of such traits is valid for further breeding programme. Similar results were also reported by Kumar et al., (2012) and Reshmika et al., (2015).

With respect to earliness parameters, minimum number of days to first flowering was noticed in HUB - 23 and Arka Nilkanth (48.00 each) while, maximum number of days to first flowering (53.00) was noticed in Acc 69, Acc- 596 and Acc- 65, with an average mean of 50.95. GV and PV were 0.55 and 1.94 respectively. Minimum number of days is preferred for earliness.

Low GCV and PCV (1.46\% and $2.74 \%$ respectively) were noticed for this trait, with low heritability and GAM $(28.4 \%$ and $1.60 \%$ respectively) along with GA (0.81). Minimum number of days to fifty $\%$ flowering (55.00) was observed in HUB - 23. GV and PV were 0.81 and 2.95 respectively. Low GCV and PCV (1.54\% and $2.94 \%$ respectively) were noticed for this trait, with low heritability and GAM (27.61 \% and $1.67 \%$ respectively) along with GA (0.97). Days to first fruit maturity for 60 genotypes ranged from 68.50 (HUB - 23). GV and PV were 4.40 and 6.75 respectively. GCV and PCV were low $(2.86 \%$ and $3.54 \%$ respectively). High heritability $(65.25 \%)$ was noticed with low GAM (4.76 $\%$ ) and GA with 3.49. The moderate PCV and GCV indicated that traits are governed by non-additive genes. Hence, there is a little scope for improvement of these traits through selection. Hence, selection for improvement of such characters will not be rewarding. These findings were in close agreement with the results obtained by Sharma and Swaroop (2000), Naik (2005), Muniappan et al., (2010), Arunkumar et al., (2013), Dahatonde et al., (2010) and Shekar et al., (2012).

Ultimate goal of the farmers is to attain high fruit yield. For fruit yield traits, IC 354597 recorded minimum fruit weight $(73.00 \mathrm{~g})$ while, maximum in Acc-597 (174.30 g) with an overall mean of $123.36 \mathrm{~g}$. GV and PV were 364.87 and 611.97 respectively. GCV (15.48 $\%)$, PCV (20.05\%) and heritability (59.62 \%) were moderate along with low GAM (24.62 $\%$ ) and GA (30.38) was noticed for this character. Number of fruits per cluster of 2.10 (Acc - 350) with an overall mean of $1.15 . \mathrm{GV}$ and PV were 0.04 and 0.05 respectively. Moderate GCV (18.42 \%) and PCV (19.84 \%) were noticed with high heritability $(86.21 \%)$, moderate GAM (35.24\%) and GA (0.40) for this trait. HUB -13 recorded maximum number of fruits per plant (21.60) while, $\mathrm{CO}-2$ with minimum number of fruits (7.40) with an overall mean of 14.99. GV and PV were 10.15 and 11.05 respectively. GCV and PCV were high with $21.24 \%$ and $22.18 \%$ respectively. There was high heritability with $91.78 \%$, moderate GAM with $41.93 \%$ and GA with 6.28 for this character studied. The variation of fruit length in the population was $7.76 \mathrm{~cm}$ (Acc-69) to $20.66 \mathrm{~cm}$ (Acc-500) with an overall mean of $11.39 \mathrm{~cm}$. GV and PV were 7.95 and 8.31 respectively. GV and PV were 7.95 and 8.31 respectively (Table $1-4$ ). 
Table.1 Analysis of variance (mean sum of squares) for growth, flowering, yield and quality parameters in brinjal

\begin{tabular}{|c|c|c|c|c|c|c|c|}
\hline $\begin{array}{l}\text { Sl. } \\
\text { No. }\end{array}$ & Source of variation/Characters & Replications & $\begin{array}{l}\text { Treatments } \\
\text { (Genotypes) }\end{array}$ & Error & S.Em \pm & $\begin{array}{c}\text { CD } \\
(5 \%)\end{array}$ & CD (1\%) \\
\hline & Degrees of freedom & 1 & 59 & 59 & & & \\
\hline 1. & Plant height $(\mathrm{cm})$ at 90 DAT & 262.55 & $69.99 *$ & 44.76 & 4.73 & 13.38 & 17.80 \\
\hline 2. & $\begin{array}{l}\text { Number of primary branches per plant at } \\
90 \text { DAT }\end{array}$ & 0.17 & $1.39 * *$ & 0.38 & 0.43 & 1.24 & 1.65 \\
\hline 3. & Stem girth $(\mathrm{cm})$ at 90 DAT & 0.09 & $0.11 * *$ & 0.04 & 0.14 & 0.4 & 0.53 \\
\hline 4. & Leaf area $\left(\mathrm{cm}^{2}\right)$ & 859.37 & $15055.02 *$ & 8282.73 & 64.35 & 182.1 & 242.24 \\
\hline 5. & Days to first flowering & 73.63 & $2.50 *$ & 1.39 & 0.83 & $2 . .36$ & 3.14 \\
\hline 6. & Days to $50 \%$ flowering & 75.20 & $3.77 * *$ & 2.14 & 1.03 & 2.92 & 3.89 \\
\hline 7. & Days to first fruit maturity & 118.00 & $11.16^{* *}$ & 2.34 & 1.08 & 3.06 & 4.08 \\
\hline 8. & Average fruit weight (g) & 251.14 & $976.85^{* *}$ & 247.10 & 11.11 & 31.45 & 41.84 \\
\hline 9. & Number of fruits per cluster & 0.07 & $0.09 * *$ & 0.007 & 0.06 & 0.16 & 0.22 \\
\hline 10. & Number of fruits per plant & 72.38 & $21.21 * *$ & 0.90 & 0.64 & 1.9 & 2.53 \\
\hline 11. & Fruit length $(\mathrm{cm})$ & 5.00 & $16.26 * *$ & 0.35 & 0.42 & 1.19 & 1.59 \\
\hline 12. & Fruit girth $(\mathrm{cm})$ & 0.05 & $0.81 * *$ & 0.26 & 0.36 & 1.02 & 1.36 \\
\hline 13. & Fruit yield per plant (kg) & 0.50 & $0.43 * *$ & 0.05 & 0.17 & 0.48 & 0.64 \\
\hline 14. & Phenol content (mg/100 g of fruit) & 53.89 & $3161.51 * *$ & 12.16 & 2.46 & 6.98 & 9.28 \\
\hline
\end{tabular}


Table.2 Estimates of mean, range, components of variance, heritability, genetic advance and genetic advance over percent of mean for growth and flowering parameters in brinjal

\begin{tabular}{|c|c|c|c|c|c|c|c|c|c|c|}
\hline $\begin{array}{l}\text { Sl. } \\
\text { No. }\end{array}$ & Character & Mean & Range & GV & PV & $\begin{array}{c}\text { GCV } \\
(\%)\end{array}$ & $\begin{array}{l}\text { PCV } \\
(\%)\end{array}$ & $\mathbf{h}^{2}$ & GA & GAM \\
\hline 1. & Plant height $(\mathrm{cm})$ at 90 DAT & 39.86 & $31.16-64.90$ & 12.61 & 57.38 & 8.91 & 19.00 & 21.98 & 3.43 & 8.60 \\
\hline 2. & $\begin{array}{l}\text { Number of primary branches } \\
\text { per plant at } 90 \text { DAT }\end{array}$ & 5.23 & $3.10-7.00$ & 0.50 & 0.89 & 13.59 & 18.05 & 56.75 & 1.10 & 21.10 \\
\hline 3. & Stem girth $(\mathrm{cm})$ at 90 DAT & 1.03 & $0.71-1.65$ & 0.03 & 0.07 & 17.80 & 26.36 & 45.61 & 0.25 & 24.77 \\
\hline 4. & Leaf area $\left(\mathrm{cm}^{2}\right)$ & 302.64 & $158.23-459.75$ & 3386.14 & 11668.88 & 19.22 & 35.69 & 29.02 & 64.57 & 21.33 \\
\hline 5. & Days to first flowering & 50.95 & $48.00-53.00$ & 0.55 & 1.94 & 1.46 & 2.74 & 28.4 & 0.81 & 1.60 \\
\hline 6. & Days to $50 \%$ flowering & 58.37 & $55.00-62.50$ & 0.81 & 2.95 & 1.54 & 2.94 & 27.61 & 0.97 & 1.67 \\
\hline 7. & Days to first fruit maturity & 73.34 & $68.50-77.50$ & 4.40 & 6.75 & 2.86 & 3.54 & 65.25 & 3.49 & 4.76 \\
\hline 8. & Average fruit weight $(\mathrm{g})$ & 123.36 & $73.00-174.30$ & 364.87 & 611.97 & 15.48 & 20.05 & 59.62 & 30.38 & 24.62 \\
\hline 9. & Number of fruits per cluster & 1.15 & $1.00-2.10$ & 0.04 & 0.05 & 18.42 & 19.84 & 86.21 & 0.40 & 35.24 \\
\hline 10. & Number of fruits per plant & 14.99 & $7.40-21.60$ & 10.15 & 11.05 & 21.24 & 22.18 & 91.78 & 6.28 & 41.93 \\
\hline 11. & Fruit length $(\mathrm{cm})$ & 11.39 & $7.76-20.66$ & 7.95 & 8.31 & 24.75 & 25.30 & 95.70 & 5.68 & 49.89 \\
\hline 12. & Fruit girth $(\mathrm{cm})$ & 4.80 & $3.06-5.73$ & 0.27 & 0.53 & 10.92 & 15.26 & 51.28 & 0.77 & 16.12 \\
\hline 13. & Fruit yield per plant (kg) & 1.83 & $0.79-2.67$ & 0.18 & 0.24 & 23.62 & 27.04 & 76.32 & 0.78 & 42.51 \\
\hline 14. & Phenols (mg/100 g) & 166.87 & $75.98-267.60$ & 1574.67 & 1586.83 & 23.78 & 23.87 & 99.23 & 81.43 & 48.79 \\
\hline $\begin{array}{l}\text { G } \\
P \\
G\end{array}$ & $\begin{array}{l}\text { Senotypic variance } \\
\text { henotypic variance } \\
\text { Genotypic co-efficient of variation }\end{array}$ & $\begin{array}{l}\mathrm{h}^{2}- \\
\text { GA- } \\
\text { GAI }\end{array}$ & $\begin{array}{l}\text { ense heritability } \\
\text { advance } \\
\text { tic advance as pe }\end{array}$ & 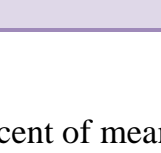 & & & & & & \\
\hline
\end{tabular}


Table.3 Per se performance of brinjal genotypes for growth and earliness parameters

\begin{tabular}{|c|c|c|c|c|c|c|c|c|c|c|}
\hline Sl. No. & Genotypes & $\begin{array}{c}\text { Plant } \\
\text { height } \\
(\mathrm{cm}) \text { at } 90 \\
\text { DAT }\end{array}$ & $\begin{array}{c}\text { No. } \\
\text { branches } \\
\text { at } 90 \text { DAT }\end{array}$ & $\begin{array}{l}\text { Stem girth } \\
\text { (cm) at } 90 \\
\text { DAT }\end{array}$ & $\begin{array}{l}\text { Leaf } \\
\text { area }(\mathrm{cm} \\
2)\end{array}$ & $\begin{array}{c}\text { Days to } \\
\text { first } \\
\text { flowering }\end{array}$ & $\begin{array}{l}\text { Days to } \\
\text { fifty \% } \\
\text { flowering }\end{array}$ & $\begin{array}{c}\text { Days to } \\
\text { first fruit } \\
\text { maturity }\end{array}$ & $\begin{array}{l}\text { No. } \\
\text { fruits } \\
\text { per } \\
\text { cluster }\end{array}$ & $\begin{array}{l}\text { No. } \\
\text { fruits } \\
\text { per } \\
\text { plant }\end{array}$ \\
\hline 1. & HUB-1 & 35.58 & 4.70 & 1.04 & 328.05 & 52.00 & 58.50 & 73.00 & 1.40 & 15.50 \\
\hline 2. & HUB-2 & 38.79 & 4.60 & 1.02 & 224.77 & 52.00 & 58.50 & 73.50 & 1.10 & 13.90 \\
\hline 3. & HUB-3 & 31.16 & 5.40 & 0.88 & 400.38 & 52.00 & 58.50 & 74.50 & 1.40 & 17.70 \\
\hline 4. & HUB-4 & 31.89 & 4.30 & 1.13 & 175.63 & 52.50 & 60.00 & 75.00 & 1.20 & 16.40 \\
\hline 5. & HUB-5 & 32.54 & 5.00 & 0.97 & 299.88 & 51.00 & 57.00 & 74.00 & 1.00 & 16.70 \\
\hline 6. & HUB-6 & 34.94 & 5.30 & 1.20 & 171.59 & 50.50 & 58.00 & 72.50 & 1.00 & 11.00 \\
\hline 7. & HUB-7 & 32.90 & 5.50 & 1.25 & 304.42 & 50.00 & 59.00 & 70.50 & 1.40 & 12.30 \\
\hline 8. & HUB-8 & 41.36 & 6.10 & 1.25 & 165.68 & 50.50 & 57.50 & 74.50 & 1.20 & 13.60 \\
\hline 9. & HUB-9 & 47.31 & 6.00 & 1.38 & 288.51 & 51.50 & 58.50 & 74.50 & 1.40 & 17.40 \\
\hline 10. & HUB-10 & 37.14 & 6.10 & 1.05 & 181.83 & 51.00 & 57.50 & 77.50 & 1.00 & 14.60 \\
\hline 11. & HUB-11 & 37.13 & 5.20 & 1.14 & 257.61 & 50.50 & 58.00 & 74.00 & 1.60 & 18.00 \\
\hline 12. & HUB-12 & 43.09 & 5.40 & 1.13 & 260.89 & 52.00 & 58.50 & 77.50 & 1.40 & 17.30 \\
\hline 13. & HUB-13 & 51.02 & 6.80 & 1.47 & 224.52 & 51.00 & 59.50 & 70.50 & 1.60 & 21.60 \\
\hline 14. & HUB-14 & 46.34 & 6.00 & 1.63 & 254.56 & 51.00 & 58.50 & 77.00 & 1.00 & 18.40 \\
\hline 15. & Acc -81 & 41.21 & 5.70 & 1.08 & 328.52 & 51.50 & 58.00 & 72.00 & 1.00 & 15.60 \\
\hline 16. & HUB-15 & 39.81 & 5.70 & 0.74 & 439.31 & 51.50 & 58.50 & 71.00 & 1.00 & 17.60 \\
\hline 17. & HUB-16 & 39.50 & 5.60 & 0.84 & 314.17 & 50.50 & 56.00 & 70.00 & 1.40 & 16.50 \\
\hline 18. & HUB-17 & 40.38 & 6.40 & 1.16 & 158.23 & 51.00 & 59.00 & 75.50 & 1.20 & 15.60 \\
\hline 19. & HUB-18 & 42.74 & 6.30 & 0.91 & 283.80 & 51.00 & 58.50 & 71.00 & 1.00 & 19.00 \\
\hline 20. & Acc-112 & 36.36 & 5.20 & 1.30 & 408.36 & 51.00 & 59.00 & 76.50 & 1.00 & 16.60 \\
\hline 21. & $\begin{array}{l}\text { Arka } \\
\text { Kusumakar }\end{array}$ & 36.31 & 5.80 & 0.94 & 296.53 & 50.50 & 58.50 & 70.00 & 1.40 & 13.70 \\
\hline 22. & HUB-19 & 40.82 & 5.90 & 1.00 & 401.87 & 51.00 & 58.50 & 75.50 & 1.00 & 12.60 \\
\hline 23. & HUB-20 & 41.55 & 6.40 & 1.39 & 304.78 & 49.00 & 56.50 & 71.00 & 1.00 & 12.00 \\
\hline 24. & HUB-21 & 42.93 & 6.10 & 1.23 & 319.92 & 50.50 & 58.00 & 74.50 & 1.00 & 18.60 \\
\hline 25. & Pusa Shymala & 44.60 & 7.00 & 1.33 & 355.83 & 51.50 & 61.00 & 74.00 & 1.00 & 19.80 \\
\hline 26. & HUB- 22 & 48.31 & 5.20 & 1.22 & 459.75 & 51.50 & 59.00 & 77.50 & 1.40 & 19.20 \\
\hline 27. & HUB- 23 & 45.02 & 6.00 & 1.17 & 255.00 & 48.00 & 55.00 & 68.50 & 1.40 & 19.10 \\
\hline 28. & Acc -359 & 42.94 & 4.60 & 0.93 & 396.89 & 50.50 & 58.50 & 72.50 & 1.20 & 17.70 \\
\hline 29. & Maritunjaya & 44.52 & 5.60 & 0.82 & 379.70 & 51.50 & 58.00 & 72.00 & 1.00 & 15.60 \\
\hline 30. & HUB-24 & 40.23 & 5.60 & 0.85 & 314.50 & 51.50 & 59.50 & 74.50 & 1.00 & 14.60 \\
\hline
\end{tabular}




\begin{tabular}{|c|c|c|c|c|c|c|c|c|c|c|}
\hline 31. & Arka Keshav & 35.40 & 5.10 & 1.16 & 292.88 & 50.50 & 59.50 & 70.00 & 1.00 & 11.90 \\
\hline 32. & Acc-355 & 38.41 & 5.90 & 0.92 & 186.05 & 50.00 & 57.50 & $72 . .00$ & 1.10 & 11.60 \\
\hline 33. & Arka Nilkanth & 43.08 & 5.70 & 1.32 & 245.45 & 48.00 & 58.50 & 69.00 & 1.40 & 12.00 \\
\hline 34. & Accession 350 & 43.15 & 5.90 & 1.03 & 204.76 & 51.00 & 58.50 & 74.50 & 2.10 & 11.00 \\
\hline 35. & $\begin{array}{l}\text { Mattigulla } \\
\text { round }\end{array}$ & 36.62 & 5.70 & 1.17 & 373.88 & 50.00 & 57.50 & 73.00 & 1.00 & 19.30 \\
\hline 36. & IC 354597 & 34.74 & 5.90 & 1.53 & 319.18 & 51.00 & 58.50 & 74.50 & 1.00 & 16.60 \\
\hline 37. & L- 3267 & 50.71 & 5.10 & 1.65 & 264.07 & 51.00 & 59.00 & 72.00 & 1.10 & 13.60 \\
\hline 38. & HUB-25 & 50.45 & 5.30 & 0.80 & 283.78 & 51.00 & 59.00 & 72.00 & 1.00 & 16.70 \\
\hline 39. & IC 326628 & 39.79 & 5.00 & 1.09 & 205.08 & 50.50 & 59.00 & 74.00 & 1.00 & 20.20 \\
\hline 40. & Acc- 113 & 64.90 & 5.30 & 0.90 & 262.98 & 49.00 & 58.50 & 70.00 & 1.00 & 16.00 \\
\hline 41. & Acc-500 & 47.06 & 4.80 & 0.78 & 422.13 & 49.00 & 59.00 & 71.00 & 1.00 & 13.50 \\
\hline 42. & Acc -596 & 44.41 & 5.40 & 0.88 & 381.92 & 52.00 & 56.00 & 75.50 & 1.00 & 13.00 \\
\hline 43. & Acc -600 & 40.22 & 4.50 & 0.82 & 321.27 & 50.00 & 58.00 & 73.00 & 1.40 & 9.50 \\
\hline 44. & Acc -65 & 43.01 & 5.70 & 0.71 & 322.14 & 52.50 & 57.50 & 74.00 & 1.00 & 10.00 \\
\hline 45. & Acc-597 & 39.82 & 4.80 & 0.78 & 387.76 & 51.00 & 58.00 & 70.50 & 1.20 & 10.20 \\
\hline 46. & Acc- 69 & 32.68 & 4.90 & 0.85 & 437.60 & 53.00 & 61.50 & 75.50 & 1.10 & 9.50 \\
\hline 47. & Acc -700 & 35.49 & 5.00 & 0.84 & 432.24 & 51.50 & 62.50 & 74.00 & 1.00 & 9.70 \\
\hline 48. & HUB-26 & 34.71 & 5.70 & 0.81 & 429.13 & 49.50 & 57.50 & 72.50 & 1.00 & 8.90 \\
\hline 49. & Acc $61-4$ & 35.01 & 4.90 & 0.86 & 169.59 & 50.50 & 56.50 & 77.50 & 1.10 & 7.40 \\
\hline 50. & Acc $61-5$ & 34.34 & 4.50 & 0.85 & 315.65 & 50.50 & 58.50 & 74.00 & 1.00 & 12.50 \\
\hline 51. & Acc -50 & 36.07 & 5.00 & 0.95 & 190.99 & 50.50 & 55.50 & 70.50 & 1.00 & 13.60 \\
\hline 52. & HUB - 27 & 37.01 & 3.80 & 0.98 & 252.76 & 50.00 & 57.00 & 71.00 & 1.10 & 16.40 \\
\hline 53. & HUB- 28 & 33.82 & 4.40 & 0.92 & 232.29 & 50.50 & 58.50 & 73.00 & 1.50 & 17.30 \\
\hline 54. & Acc 61-2 & 35.29 & 3.50 & 0.77 & 179.20 & 52.50 & 61.00 & 76.00 & 1.00 & 14.10 \\
\hline 55. & Acc - 51 & 34.91 & 3.50 & 0.85 & 363.29 & 52.50 & 58.50 & 76.50 & 1.20 & 15.10 \\
\hline 56. & Acc -10 & 39.08 & 4.20 & 0.80 & 453.44 & 52.50 & 60.00 & 75.50 & 1.10 & 19.80 \\
\hline 57. & HUB- 29 & 37.44 & 4.50 & 0.77 & 445.10 & 52.00 & 59.50 & 74.50 & 1.00 & 17.90 \\
\hline 58. & HUB- 30 & 36.13 & 3.60 & 0.79 & 372.28 & 53.00 & 59.50 & 76.50 & 1.00 & 13.90 \\
\hline 59. & HUB-31 & 35.06 & 3.70 & 0.78 & 259.20 & 49.50 & 55.50 & 70.00 & 1.00 & 16.00 \\
\hline \multirow[t]{5}{*}{60.} & Acc -65 & 35.07 & 3.10 & 0.95 & 196.91 & 53.00 & 58.00 & 74.00 & 1.00 & 14.20 \\
\hline & C.V & 16.78 & 11.87 & 19.44 & 19.07 & 2.31 & 2.50 & 2.08 & 4.36 & 6.35 \\
\hline & SE.m & 4.73 & 0.43 & 0.14 & 64.35 & 0.83 & 1.03 & 1.08 & 0.06 & 0.67 \\
\hline & $\mathrm{CD}$ at $5 \%$ & 13.38 & 1.24 & 0.40 & 182.10 & 2.36 & 2.92 & 3.06 & 0.16 & 1.90 \\
\hline & $\mathrm{CD}$ at $1 \%$ & 17.80 & 1.65 & 0.53 & 242.24 & 3.14 & 3.89 & 4.07 & 0.22 & 2.53 \\
\hline
\end{tabular}


Table.4 Per se performance of yield and quality parameters of brinjal genotypes

\begin{tabular}{|c|c|c|c|c|c|c|}
\hline SI. No. & Genotypes & $\begin{array}{l}\text { Average fruit } \\
\text { weight (g) }\end{array}$ & $\begin{array}{c}\text { Fruit yield } \\
\text { per plant } \\
\text { (kg) }\end{array}$ & $\begin{array}{l}\text { Fruit } \\
\text { length } \\
\text { (cm) }\end{array}$ & $\begin{array}{c}\text { Fruit } \\
\text { girth }(\mathrm{cm})\end{array}$ & $\begin{array}{c}\text { Phenols } \\
\text { (mg/100 } \\
\text { g) }\end{array}$ \\
\hline 1. & HUB-1 & 140.70 & 2.18 & 9.83 & 5.38 & 140.95 \\
\hline 2. & HUB-2 & 130.40 & 1.81 & 10.22 & 4.83 & 179.83 \\
\hline 3. & HUB-3 & 131.60 & 2.32 & 10.49 & 4.97 & 206.96 \\
\hline 4. & HUB-4 & 131.80 & 2.16 & 10.12 & 5.05 & 167.10 \\
\hline 5. & HUB-5 & 139.90 & 2.30 & 9.15 & 4.84 & 107.55 \\
\hline 6. & HUB-6 & 152.00 & 1.66 & 10.68 & 5.10 & 196.50 \\
\hline 7. & HUB-7 & 144.60 & 1.76 & 8.85 & 4.77 & 165.60 \\
\hline 8. & HUB-8 & 154.10 & 2.08 & 10.43 & 5.65 & 216.95 \\
\hline 9. & HUB-9 & 137.80 & 2.38 & 11.76 & 4.90 & 134.60 \\
\hline 10. & HUB-10 & 149.60 & 2.18 & 8.95 & 5.36 & 173.05 \\
\hline 11. & HUB-11 & 128.20 & 2.30 & 10.41 & 4.96 & 199.00 \\
\hline 12. & HUB-12 & 150.80 & 2.56 & 10.47 & 4.88 & 133.50 \\
\hline 13. & HUB-13 & 123.80 & 2.67 & 10.22 & 5.35 & 155.10 \\
\hline 14. & HUB-14 & 111.40 & 2.03 & 9.65 & 5.05 & 84.58 \\
\hline 15. & Acc -81 & 122.70 & 1.90 & 10.56 & 4.95 & 174.00 \\
\hline 16. & HUB-15 & 122.40 & 2.14 & 8.58 & 5.20 & 194.50 \\
\hline 17. & HUB-16 & 126.20 & 2.05 & 9.19 & 4.60 & 242.03 \\
\hline 18. & HUB-17 & 126.30 & 1.97 & 9.68 & 5.30 & 235.45 \\
\hline 19. & HUB-18 & 104.00 & 2.02 & 10.00 & 5.01 & 166.00 \\
\hline 20. & Acc-112 & 96.20 & 1.59 & 8.80 & 5.14 & 147.60 \\
\hline 21. & Arka Kusumakar & 141.20 & 1.92 & 9.70 & 4.62 & 193.40 \\
\hline 22. & HUB-19 & 124.00 & 1.56 & 11.27 & 4.75 & 216.05 \\
\hline 23. & HUB-20 & 109.90 & 1.32 & 11.41 & 4.12 & 206.05 \\
\hline 24. & HUB-21 & 117.20 & 2.18 & 10.50 & 3.66 & 191.00 \\
\hline 25. & Pusa Shymala & 93.60 & 1.85 & 17.10 & 3.10 & 267.60 \\
\hline 26. & HUB- 22 & 123.60 & 2.36 & 11.92 & 3.86 & 165.95 \\
\hline 27. & HUB- 23 & 121.80 & 2.32 & 12.31 & 4.62 & 203.65 \\
\hline 28. & Acc - 359 & 105.90 & 1.86 & 12.46 & 4.28 & 166.05 \\
\hline 29. & Maritunjaya & 117.40 & 1.83 & 13.32 & 5.48 & 147.25 \\
\hline 30. & HUB-24 & 102.10 & 1.48 & 18.44 & 4.70 & 217.61 \\
\hline
\end{tabular}


Int.J.Curr.Microbiol.App.Sci (2019) 8(7): 2234-2244

\begin{tabular}{|c|c|c|c|c|c|c|}
\hline 31. & Arka Keshav & 89.20 & 1.06 & 14.80 & 3.06 & 185.53 \\
\hline 32. & Acc-355 & 86.00 & 0.99 & 12.20 & 4.29 & 146.23 \\
\hline 33. & Arka Nilkanth & 122.80 & 1.46 & 16.46 & 4.79 & 167.61 \\
\hline 34. & Accession 350 & 73.20 & 0.79 & 11.26 & 4.49 & 144.97 \\
\hline 35. & Mattigulla round & 107.90 & 2.07 & 9.72 & 4.75 & 236.10 \\
\hline 36. & IC 354597 & 73.00 & 1.21 & 10.58 & 3.69 & 75.98 \\
\hline 37. & L- 3267 & 111.50 & 1.51 & 15.03 & 3.31 & 115.06 \\
\hline 38. & HUB-25 & 90.10 & 1.50 & 14.44 & 5.19 & 126.56 \\
\hline 39. & IC 326628 & 131.40 & 2.64 & 19.39 & 4.83 & 145.23 \\
\hline 40. & Acc- 113 & 74.30 & 1.16 & 14.86 & 3.30 & 170.56 \\
\hline 41. & Acc -500 & 153.20 & 2.06 & 20.66 & 3.62 & 133.26 \\
\hline 42. & Acc -596 & 157.20 & 2.04 & 16.97 & 4.38 & 175.55 \\
\hline 43. & Acc -600 & 92.20 & 0.87 & 12.40 & 4.68 & 125.48 \\
\hline 44. & Acc -65 & 136.90 & 1.37 & 14.21 & 5.35 & 119.12 \\
\hline 45. & Acc-597 & 174.30 & 1.82 & 11.07 & 5.24 & 165.11 \\
\hline 46. & Acc- 69 & 131.80 & 1.25 & 7.76 & 5.04 & 187.39 \\
\hline 47. & Acc -700 & 100.80 & 0.98 & 9.16 & 5.23 & 113.12 \\
\hline 48. & HUB-26 & 138.90 & 1.24 & 8.94 & 4.95 & 203.12 \\
\hline 49. & Acc 61-4 & 135.10 & 1.00 & 11.76 & 5.06 & 143.62 \\
\hline 50. & Acc 61-5 & 138.40 & 1.73 & 9.09 & 5.28 & 184.51 \\
\hline 51. & Acc -50 & 150.70 & 2.04 & 10.98 & 5.33 & 166.11 \\
\hline 52. & HUB - 27 & 142.70 & 2.32 & 9.37 & 5.43 & 126.12 \\
\hline 53. & HUB- 28 & 136.10 & 2.34 & 9.72 & 5.29 & 111.52 \\
\hline 54. & Acc 61-2 & 116.40 & 1.64 & 10.29 & 4.92 & 166.22 \\
\hline 55. & Acc - 51 & 134.80 & 2.03 & 8.95 & 5.73 & 148.11 \\
\hline 56. & Acc -10 & 117.90 & 2.34 & 9.58 & 5.22 & 177.52 \\
\hline 57. & HUB- 29 & 110.10 & 1.96 & 8.39 & 5.36 & 166.02 \\
\hline 58. & HUB- 30 & 131.60 & 1.83 & 9.63 & 5.18 & 93.48 \\
\hline 59. & HUB-31 & 142.80 & 2.28 & 10.36 & 5.40 & 207.46 \\
\hline \multirow[t]{5}{*}{60.} & Acc -65 & 119.50 & 1.70 & 9.11 & 5.42 & 190.05 \\
\hline & C.V & 3.95 & 13.16 & 5.24 & 10.65 & 2.09 \\
\hline & SE.m & 11.11 & 0.17 & 0.42 & 0.36 & 2.46 \\
\hline & $\mathrm{CD}$ at $5 \%$ & 31.45 & 0.48 & 1.19 & 1.02 & 6.98 \\
\hline & $\mathrm{CD}$ at $1 \%$ & 41.84 & 0.64 & 1.59 & 1.36 & 9.28 \\
\hline
\end{tabular}


GCV (24.75\%) and PCV (25.30\%) were high, coupled with high heritability of 95.70 $\%$, along with moderate GAM (49.89\%) and GA with 5.68 was observed for this trait. The high PCV and GCV indicated that traits were governed by additive genes. Hence, there is more scope for improvement of these traits through selection. Several workers like Dahatonde et al., (2010), Das et al., (2010), Muniappan et al., (2010), Kumar et al., (2012), Arunkumar et al., (2013), Lokesh et al., (2013), Nilakh et al., (2017), Tripathy et al., (2017) and Verma et al., (2018) reported similar findings.

Fruit girth of $3.06 \mathrm{~cm}$ (Arka Keshav) with an overall mean of $4.80 \mathrm{~cm} . \mathrm{GV}$ and PV were 0.27 and 0.53 was noticed respectively. Moderate GCV (10.92 \%) and medium PCV $(15.26 \%)$ coupled with moderate heritability with $51.28 \%$, low GAM with $16.12 \%$ and GA with 0.77 were noticed for this character. The variation in the fruit yield per plant in the population was $0.79 \mathrm{~kg}$ (Acc-350) to $2.67 \mathrm{~kg}$ (HUB - 13) with an overall mean of $1.83 \mathrm{~kg}$. High GCV (23.62 \%) and PCV (27.04 \%) coupled with high heritability $(76.32 \%)$ was noticed for this trait along with moderate GAM (42.51\%) and GA with 0.78. Among the genotypes studied, phenols ranged from 75.98 (IC 354597) to 267.60 (Pusa Shymala) with an overall mean of $166.87(\mathrm{mg} / 100 \mathrm{~g})$. GV and PV were 1574.67 and 1586.83 respectively. GCV and PCV were high (23.78 $\%$ and $23.87 \%$ respectively) coupled with high heritability (99.23 \%), along with moderate GAM (48.79\%) and GA with 81.43 were observed for this trait. The moderate PCV and GCV indicated that these traits were governed by non additive genes. Hence, there is a little scope for improvement of these traits through selection. Hence, selection for improvement of such characters will not be rewarding. Similar results were also reported by Kumar et al., (2012) and Reshmika et al., (2015). From this research findings, it was concluded that there was existence of sufficient variability in genetic materials studied and the environmental role is negligible. Hence, there is ample of scope for improving the yield characters with direct selection for further breeding programme.

\section{References}

Arunnkumar, B., Kumar, S. V. S. and Prakash, C. G., 2013, Genetic variability and divergence studies in brinjal (Solanum melongena L.). Bioinfolet, 10(2B): 739-744.

Dahatonde, K., Dod, V. N., Nagare, P. K. and Wag A. P., 2010, Correlation and path analysis studies in purple fruited brinjal (Solanum melongena L.). Asian J. Hort., 5 (2): 428-430.

Das, S., Mandal, A. B. and Hazra, P., 2010, Study of heterosis in brinjal (Solanum melongena L.) for yield attributing traits. J. of Crop and Weed, 5 (2): 2530.

Kumar, S. R., Arumugam, T. and Premalakshmi, V., 2012, Evaluation and variability studies in local types of brinjal for yield and quality (Solanum melongena L.). Electronic J. Plant Breed, 3 (4): 977-982.

Lokesh, B., Reddy, P. S., Reddy, R. V. S. K. and Sivaraj, N., 2013, Variability, heritability and genetic advance studies in brinjal (Solanum melongena L.). Electronic J. Pl. Breed., 4(1): 10971100.

Muniappan, S., Saravanan, K. and Ramya, B., 2010, Studies on genetic divergence and variability for certain economic characters in eggplant (Solanum melongena L.). Electronic J. Plant Breed, 1 (4): 462-465.

Naik, A., Kumar, S. and Yadav, Y. C., 2005, Variability studies for yield and yield attributing characters in brinjal (Solanum melongena L.). Prog. Agric., 
11(2): 486-488.

Nilakh, S. B., Thaware, B. L., Dhekale, J. S. Palshetkar, and M. G. 2017. Genetic variability studies on f5 generation of brinjal (Solanum melongena L.). Plant Archives. 17 (1): 103-105

Reshmika, P. K., Gasti, V. D., Evoor, S., Jayappa, and Mulge, R. 2015, Genetic variability studies for growth, earliness, yield and quality parameters in brinjal (Solanum melongena L.). Environ \& Ecol., 33 (2): 761-766.

Sharma and Swaroop (2000), Sharma, T. V. R. S. and. Swaroop, K., 2000, Genetic variability and character association in brinjal (Solanum melongena L.). Indian J. Hortic., 57(10): 59-65.
Shekar, K, C., Ashok, P., Sasikala, K., 2012, Studies on heritability and multivariate analyses in brinjal (Solanum melongena L.). Veg. Crp. Res. Bulletin, 76: 79-88.

Tripathy, B., Sharma,D., Jangde, B. and Pappu, L. B., 2017, Genetic variability and heritability studies in brinjal (Solanum melongena L.). The Biosc., 10: 109-106.

Verma, P., Kushwaha M. L. and Ankit P. 2018, Studies on Variability, heritability and genetic advance for yield attributing traits in brinjal (Solanum melongena L.) for two different seasons. Int.J.Curr.Microbiol.App.Sci. $\quad$ 7(9): 1543-1552.

\section{How to cite this article:}

Shruti, P.G., C.N. Hanchinamani, N. Basavaraja, M.S. Kulkarni, S. Cholin and Tatagar, M.H. 2019. Genetic Variability Studies for Yield and Yield Attributing Traits in Brinjal (Solanum melongena L.). Int.J.Curr.Microbiol.App.Sci. 8(07): 2234-2244.

doi: https://doi.org/10.20546/ijcmas.2019.807.272 